\title{
Diferencias en la composición corporal y actividad física en estudiantes universitarios según año de ingreso
}

\author{
Differences in body composition and physical activity in university students according to the year of admission
}

\author{
Fernando Rodríguez-Rodríguez ${ }^{*}$, Mitzi Santibañez-Miranda², Grundy Montupin-Rozas ${ }^{3}$, Francisca Chávez-Ramírez ${ }^{4}$, \\ Patricio Solis-Urra ${ }^{5}$
}

1 Profesor de Educación Física. Grupo IRyS, Escuela de Educación Física, Pontificia Universidad Católica de Valparaíso. Valparaíso, Chile. e-mail: fernando.rodriguez@pucv.cl ORCID: 0000-0002-4999-4857

2 Nutricionista, Licenciada en Nutrición. Centro Médico MEDIGROUP. Santiago, Chile. e-mail: m.santibanez.miranda@gmail.com

3 Nutricionista, Licenciada en Nutrición y Dietética. Valparaíso, Chile. e-mail: grundyliz@hotmail.com

4 Nutricionista, Licenciada en Nutrición y Dietética. Salud y vida. Valparaíso, Chile. e-mail: francisca.ch@hotmail.es

5 Profesor de Educación Física. Grupo IRyS, Escuela de Educación Física, Pontificia Universidad Católica de Valparaíso. Valparaíso, Chile. e-mail: patricio.solis@pucv.cl ORCID 0000-0002-2493-9528

Fecha de recepción: Marzo 24 - 2016 Fecha de revisión: Noviembre 21 - $2016 \quad$ Fecha de aceptación: Diciembre 18 - 2016

Rodríguez-Rodríguez F, Santibañez-Miranda M, Montupin-Rozas G, Chávez-Ramírez F, Solis-Urra P. Diferencias en la composición corporal y actividad física en estudiantes universitarios según año de ingreso. Univ. Salud. 2016;18(3):474-481. DOI: http://dx.doi.org/10.22267/rus.161803.52

\section{Resumen}

Introducción: La etapa universitaria es un proceso determinante para consolidar los hábitos de vida, donde los estudiantes se ven obligados a cambiar la mayoría de sus hábitos, que propician una mala alimentación, pobre organización del tiempo y menor actividad física, teniendo repercusiones en la condición y estructura física, lo que puede afectar considerablemente el estado de salud de esta población. Objetivo: El objetivo de este trabajo fue determinar las diferencias en la composición corporal y actividad física en estudiantes universitarios según su año de ingreso. Materiales y métodos: Participaron en el estudio 287 estudiantes de ambos sexos (171 mujeres y 116 hombres), pertenecientes a las facultades de "Filosofía y Educación", "Ingenierías" y "Ciencias", a los cuales se les realizó una evaluación antropométrica para estimar la composición corporal con las ecuaciones de De Rose y Guimaraes y un cuestionario de frecuencia de actividad física. Resultados: Los resultados indican que existen diferencias en el Índice de Masa Corporal y masa grasa entre estudiantes mujeres de $1^{\circ}$ y $2^{\circ}$ año y entre $1^{\circ}$ y $5^{\circ}$ año en los hombres. La masa muscular no presenta grandes diferencias entre los grupos, pero si se aprecia cambios negativos entre el Índice de Masa Corporal y masa grasa de estudiantes que recién ingresan versus los estudiantes de último año. Conclusiones: Durante la universidad se vive un periodo crítico que afecta el nivel actividad física y el estado nutricional de los estudiantes y que podría empeorar en estudiantes con más años en la universidad.

Palabras clave: Universitarios; composición corporal; actividad física. (Fuente: DeCS, Bireme).

\begin{abstract}
Introduction: The university stage is a determining process when consolidating people's habits of life, where students are forced to change most of their habits favoring a poor diet, poor organization of time and less physical activity, which at the same time, has an impact on the condition and physical structure and can significantly affect the health
\end{abstract}


status of this population. Objective: To determine the differences in body composition and physical activity in university students according to their year of admission. Materials and methods: The study involved 287 male and female students (171 women and 116 men) who belonged to the faculties of Philosophy and Education, Engineering and Science, who underwent an anthropometric assessment to estimate body composition with De Rose and Guimaraes equations and a frequency of physical activity questionnaire. Results: The results show that there are differences in Body Mass Index and fat mass among female students of first and second year and between the first and fifth year in men. Muscle mass had no significant differences between groups, but there is negative changes between Body Mass Index and fat mass from students newly admitted versus students in their last year. Conclusions: During the university stage, students face a critical period that affects their physical activity level and nutritional status, which could get worse for students with more years at the university.

Keywords: University students; body composition; motor activity. (Source: DeCS, Bireme).

\section{Introducción}

En Chile, el segmento universitario constituye uno de los grupos objetivos de la política de promoción de salud desde hace varios años. En este ámbito, se plantea como objetivo "promover cambios en la situación de salud del país a través de las generaciones de estudiantes que egresan de las universidades e institutos de educación superior, es una forma efectiva de fomentar cambios a favor del bienestar y la salud en las instituciones y comunidades en las que trabajarán"1,2. Los hábitos poco saludables, provocan una serie de consecuencias que perjudican el estado de salud de las personas y finalmente influyen en su calidad de vida ${ }^{3}$.

En Chile la población con exceso de peso creció de un $61 \%$ a un $67 \% \%^{4}$, destacando de esta última que el 37,8\%, representa a los jóvenes entre 15 y 24 años, cifra que es elevada, pero no lo suficiente como para transformarse en un importante factor de riesgo cardiovascular, pues es menor que el promedio nacional, pese a que en términos epidemiológicos se cuente con los antecedentes que permitan predecir la creciente evolución de estos indicadores, hacia el exceso de peso incluso en universitarios ${ }^{5}$.

En el contexto universitario existen factores psicológicos, sociales, culturales y económicos ${ }^{6}$, que asociados al elevado sedentarismo, pueden afectar la composición corporal de este segmento de la población. La etapa universitaria es un proceso determinante al momento de consolidar los hábitos de vida, por lo tanto, es una etapa en la cual los estudiantes se ven obligados a cambiar la mayoría de sus hábitos. Muchos salen del hogar de sus padres para mudarse cerca de las universidades, pasando a una vida parcialmente independiente, que en consecuencia, propicia una mala alimentación, descoordinación en la organización de los tiempos y el descuido en la realización de actividad física, teniendo repercusiones en la condición y estructura física, a futuro podría afectar la salud de esta población 7 .

La ingesta alimentaria, tanto en cantidad como en calidad, sumado a la poca o nula actividad física realizada por este grupo y por la población general, son los principales factores que podrían influir en la composición corporal, presentando un aumento de masa grasa y una disminución de la masa muscular, entendiendo que a mayor nivel de actividad física, menores niveles de grasa corporal, y menor actividad física, mayores niveles de grasa corporal ${ }^{8}$.

El objetivo de este estudio es determinar las diferencias que existen en la composición corporal y actividad física, según año de ingreso a la universidad en estudiantes de la Pontificia Universidad Católica de Valparaíso, de las facultades de "Filosofía y Educación", "Ingeniería" y “Ciencias", de la V región.

\section{Materiales y métodos}

Este estudio es de tipo descriptivo, comparativo y correlacional, considerando una muestra de participantes no alaeatoria e intencionada.

La muestra estuvo conformada por 287 estudiantes voluntarios de ambos sexos, entre ellos 171 mujeres y 116 hombres, pertenecientes 
a la Pontificia Universidad Católica de Valparaíso, de las facultades de "Filosofía y Educación", "Ingeniería" y "Ciencias", correspondientes a 37 diferentes carreras y que asisten a la asignatura
"Autocuidado y Vida Saludable", que reúne a estudiantes ingresados entre los años 2009 y 2013 (Tabla 1).

Tabla 1. Distribución de muestra en estudio

\begin{tabular}{|c|c|c|c|c|c|c|c|c|c|c|c|c|}
\hline \multirow[t]{2}{*}{ Año de ingreso } & \multicolumn{2}{|c|}{10} & \multicolumn{2}{|c|}{$2^{\mathrm{o}}$} & \multicolumn{2}{|c|}{$3^{\circ}$} & \multicolumn{2}{|c|}{ 4⿳⺈ } & \multicolumn{2}{|c|}{$5^{\mathbf{o}}$} & \multicolumn{2}{|c|}{ Total } \\
\hline & $\mathbf{N}^{\circ}$ & $\%$ & $\mathbf{N}^{\circ}$ & $\%$ & $\mathbf{N}^{\circ}$ & $\%$ & $\mathbf{N}^{\circ}$ & $\%$ & $\mathbf{N}^{\circ}$ & $\%$ & $\mathbf{N}^{\circ}$ & $\%$ \\
\hline Mujeres & 30 & 54,5 & 23 & 57,5 & 56 & 84,8 & 30 & 56,6 & 32 & 43,8 & 171 & 59,6 \\
\hline Hombres & 25 & 45,5 & 17 & 42,5 & 10 & 15,2 & 23 & 43,4 & 41 & 56,2 & 116 & 40,4 \\
\hline Total & 55 & 19,2 & 40 & 13,9 & 66 & 23 & 53 & 18,5 & 73 & 25,4 & 287 & 100 \\
\hline
\end{tabular}

Se aplicó el cuestionario CASEDU (Calidad del Servicio Deportivo Universitario) validado para población universitaria y obtenido del estudio de Espinoza y colaboradores del 2011'11. Este fue aplicado en formato piloto y validado nuevamente por expertos, donde se hicieron las correcciones y ajustes para perfeccionar el instrumento.

El cuestionario contiene 8 preguntas de respuesta cerrada, las cuales son: ¿Realiza ejercicio en la semana?, ¿Qué actividad física realiza?, ¿Por qué motivos realiza actividad física?, ¿En qué lugar o espacio físico realiza ejercicio?, Si no realiza actividad física ¿Por qué razón?, ¿Su casa de estudio le proporciona un espacio físico para realizar deporte?, Si la respuesta anterior fue no ¿le gustaría que la universidad contara con un espacio físico para realizar deporte?, ¿Le gustaría que su Universidad incluyera asignaturas obligatorias con créditos que promuevan el ejercicio físico?

Para la evaluación de los componentes corporales, se utilizó una balanza mecánica TANITA (precisión de 50 g), un Tallímetro de pared portátil SECA (cap. máx. $220 \mathrm{Kg}$ y $200 \mathrm{~cm}$ y precisión $1 \mathrm{~mm}$ ), una cinta métrica metálica ROSSCRAFT para perímetros, un antropómetro corto para diámetros óseos y un caliper o plicómetro para pliegues cutáneos, ambos instrumentos modelo Gaucho Pro de Rosscraft.

Se aplica el consentimiento informado que explica los procedimientos y los aspectos éticos del estudio. Luego se aplica el cuestionario de actividad física CASEDU, para finalmente realizar la evaluación antropométrica que consideró el peso, estatura, 4 pliegues (tríceps, subescapular, suprailiaco y abdominal) y 2 diámetros (estiloideo y bicondileo del fémur). Para la estimación de los resultados de la composición corporal se utilizó la estrategia de "De Rose y Guimaraes"9. El protocolo y los puntos de medición antropométrica, son definidos por las recomendaciones de Norton y Olds ${ }^{10}$.

Los sujetos voluntarios fueron evaluados por la mañana durante las clases, bajo condiciones normales de temperatura y humedad. Este procedimiento lo realiza un equipo de profesionales de Educación Física de la Escuela de Educación Física de la Pontificia Universidad Católica de Valparaíso, acreditados para evaluación antropométrica, y estudiantes de último año de Nutrición y Dietética de la Universidad de Playa Ancha.

Se utilizó la prueba de comparación de medias Tstudent, considerando un valor significativo de $\mathrm{p}<$ 0,05 para valores de igual varianza, tanto para variables continuas (composición corporal) y categóricas (Cuestionario CASEDU). El análisis se realizó en el programa Excel 2010.

\section{Consideraciones éticas}

Antes de realizar las evaluaciones, todos los sujetos firmaron un consentimiento informado que explica los procedimientos que se realizarían y que la participación en el estudio es voluntaria y 
los datos serían utilizados de manera anónima. La autorización del estudio fue otorgada por la Dirección de Investigación de la Pontificia Universidad Católica de Valparaíso y fue catalogada como investigación sin riesgo.

\section{Resultados}

De los 287 alumnos estudiados, un $62,4 \%$ se encuentra en un estado nutricional normal, un $34,9 \%$ por malnutrición por exceso y un $2,8 \%$ se encuentra en malnutrición por déficit.

El 71,3\% de las mujeres evaluadas se encuentran en peso normal, $19,9 \%$ con sobrepeso, $5,8 \%$ presenta obesidad y un $2,9 \%$ en estado de desnutrición. Las mujeres de 1 er año presentan el menor porcentaje en estado nutricional normal con un $47,8 \%$ y las de $5^{\circ}$ año, el $84,4 \%$ tienen peso normal. Inversamente, el grupo que presenta mayores niveles de desnutrición es ler año con un $6,7 \%$, mientras que los estudiantes de $2^{\circ}$ y $5^{\circ}$ año, no presentan algún tipo de desnutrición por déficit.

\section{Composición corporal}

El $49,1 \%$ de los hombres, presentan un IMC en rango de normalidad, el 41,4\% sobrepeso, 6,9\%, obesidad, y el 2,6\% presenta desnutrición. Los hombres estudiados que presentan porcentajes inferiores de sobrepeso y obesidad en comparación a sus pares mujeres.

En la tabla 2, se aprecia una diferencia significativa en el IMC entre las mujeres de $1^{\circ}$ y $2^{\circ}$ año $(\mathrm{p}=0,000)$. Respecto de los hombres se observa que los estudiantes de $5^{\circ}$ año tienen un mayor IMC que el resto de los grupos $(2013 \mathrm{p}=$ 0,036; $2012 \mathrm{p}=0,024 ; 2011 \mathrm{p}=0,068 ; 2010$ $\mathrm{p}=0,044)$.

Respecto a la composición corporal, se presentan los valores de los cuatro componentes de la composición corporal, considerando como más relevantes, la masa grasa y masa muscular. Las mujeres de $2^{\circ}$ año tienen significativamente más masa grasa que las estudiantes de $1^{\text {o }}$ año $(\mathrm{p}=0,000)$. En el caso de los hombres la masa grasa va aumentando de acuerdo a los años de ingreso a la universidad, hasta volverse significativamente más alto en estudiantes de $5^{\circ}$ año, respecto de $1^{\circ}(\mathrm{p}=0,019)$ y $2^{\circ}$ año $(\mathrm{p}=0,001)$. La masa muscular de las mujeres de $3^{\circ}(p=0,010)$ y $4^{\circ}$ año $(p=0,008)$ es significativamente más baja que los otros grupos. En los hombres no existen diferencias significativas en la masa muscular entre los distintos grupos.

\section{Actividad física}

Del total de 287 estudiantes, 210 realizan algún tipo de ejercicio. De las mujeres sólo un 64,9\% realizan alguna actividad física y mientras los hombres el valor es mayor con un 85,3 \%. Las actividades físicas realizadas varían desde artes marciales, hasta las más comunes como el running, caminata y otras populares como el futbol en hombres y la zumba en las mujeres. Dentro del espectro de ejercicios que realizan estos estudiantes, también se encuentran los ejercicios realizados con máquinas de pesos, trabajo en elípticas, trotadoras y pesas.

Del total de los alumnos que realizan actividad física, el $62 \%$ corresponde a aquellos que practican actividad física "A veces" (mujeres= 59,6\%; hombres $=65,5 \%$ ). El porcentaje de alumnos que realiza actividad física de 1 a 3 veces por semana aumenta conforme avanzan los años, con un $54,5 \%$ en $1^{\circ}$ año y $67,1 \%$ en $5^{\circ}$ año.

La actividad física "Frecuentemente", lo realiza el $10,1 \%$ de los estudiantes (mujeres= 4,7\%; hombres $=18,1 \%$ ), siendo los estudiantes de $4^{\circ}$ año, quienes realizan más frecuentemente actividad física (17\%) en comparación a los estudiantes de $2^{\circ}(0 \%)$.

La opción "Siempre" mantiene el mínimo de preferencia, con cifras menores al 3\% en los estudiantes de $2^{\circ}, 3^{\circ}$ y $4^{\circ}$ año, sin evidenciar estudiantes que realicen actividad física diariamente en $1^{\circ}$ y $5^{\circ}$ años.

Se destaca que la respuesta "Nunca" es más frecuente en mujeres, mientras que la respuesta "Siempre" y "Frecuentemente" tienden a ser mayores en hombres. En general, son los hombres quienes practican más actividad física que las mujeres (Figura 1). 
Tabla 2. Composición corporal, diferenciados por año y sexo (Promedios; Desviación estándar: DS)

\begin{tabular}{|c|c|c|c|c|c|c|c|c|c|}
\hline & \multicolumn{9}{|c|}{ Hombres } \\
\hline & & \multicolumn{2}{|l|}{$1^{\mathrm{o}}$} & $2^{o}$ & \multicolumn{2}{|l|}{$3^{\mathrm{o}}$} & $4^{\circ}$ & \multicolumn{2}{|l|}{$5^{o}$} \\
\hline & & & DS & \pm DS & \pm 1 & DS & \pm DS & \pm & DS \\
\hline IMC & $\mathrm{kg} / \mathrm{m}$ & a $24,5 \pm$ & 3,1 & a $23,7 \pm 4,5$ & a $24,6 \pm 3$ & 3,0 & a $24,6 \pm 3,2$ & $25,9 \pm$ & 3,1 \\
\hline \multirow{2}{*}{ Masa grasa } & $\mathrm{Kg}$ & d $13,3 \pm$ & 5,1 & d $11,8 \pm 4,6$ & $13,9 \pm$ & 5,2 & $14,7 \pm 4,4$ & $16,1+$ & 5,1 \\
\hline & $\%$ & $17,6 \pm$ & 5,0 & $16,8 \pm 3,1$ & $18,1 \pm$ & 4,3 & $19,6 \pm 3,9$ & $20,1 \pm$ & 4,4 \\
\hline \multirow{2}{*}{ Masa ósea } & $\mathrm{Kg}$ & $8 \pm$ & 0,9 & $7,1 \pm 0,8$ & $8 \pm 1$ & 1,0 & $8 \pm 0,7$ & $8,1 \pm$ & 1,6 \\
\hline & $\%$ & $10,9 \pm$ & 1,6 & $10,8 \pm 1,9$ & $10,8 \pm$ & 1,5 & $11 \pm 1,5$ & $10,4 \pm$ & 2,0 \\
\hline \multirow{2}{*}{ Masa residual } & $\mathrm{Kg}$ & $17,8 \pm$ & 2,1 & $16,4 \pm 3,4$ & $18,2 \pm 3$ & 3,1 & $17,9 \pm 2,4$ & $18,9 \pm$ & 2,9 \\
\hline & $\%$ & $24,1 \pm$ & 0,0 & $24,1 \pm 0,0$ & $24,1 \pm$ & 0,0 & $24,1 \pm 0,0$ & $24,1 \pm$ & 0,0 \\
\hline \multirow{2}{*}{ Masa muscular } & $\mathrm{Kg}$ & $34,8 \pm$ & 3,4 & $32,7 \pm 6,1$ & $35,3 \pm$ & 5,9 & $33,6 \pm 4,4$ & $35,4 \pm$ & 4,7 \\
\hline & $\%$ & $47,4 \pm$ & 4,2 & $48,3 \pm 1,7$ & $46,9 \pm$ & 3,6 & $45,3 \pm 3,5$ & $45,4 \pm$ & 3,9 \\
\hline & \multicolumn{9}{|c|}{ Mujeres } \\
\hline IMC & $\mathrm{kg} / \mathrm{m}$ & b $22,3 \pm$ & 3,0 & $25,4 \pm 3,1$ & $23,3 \pm 3$ & 3,3 & $23,5 \pm 3,2$ & $23 \pm$ & 3,0 \\
\hline \multirow{2}{*}{ Masa grasa } & $\mathrm{Kg}$ & $11,3 \pm$ & 3,2 & c $15,6 \pm 5,0$ & c $13,5 \pm$ & 4,2 & c $14,2 \pm 4,8$ & $12,6 \pm$ & 4,0 \\
\hline & $\%$ & $19,2 \pm$ & 3,1 & $23,5 \pm 4,5$ & $22,4 \pm$ & 4,0 & $22,9+4,8$ & $20,9 \pm$ & 4,2 \\
\hline \multirow{2}{*}{ Masa ósea } & $\mathrm{Kg}$ & $6 \pm$ & 0,6 & $6,1 \pm 0,5$ & $6 \pm$ & 0,6 & $6,3+0,8$ & $6,2 \pm$ & 0,7 \\
\hline & $\%$ & $10,5 \pm$ & 1,0 & $9,5 \pm 1,1$ & $10,2 \pm$ & 1,0 & $10,5 \pm 1,2$ & $10,5 \pm$ & 1,1 \\
\hline \multirow{2}{*}{ Masa residual } & $\mathrm{Kg}$ & $12,1 \pm$ & 1,6 & $13,6 \pm 1,9$ & $12,3 \pm 2$ & 2,0 & $12,7 \pm 2,0$ & $12,4 \pm$ & 1,6 \\
\hline & $\%$ & $20,9 \pm$ & 0,0 & $20,9 \pm 0,0$ & $20,9 \pm$ & 0,0 & $20,9 \pm 0,0$ & $20,9 \pm$ & 0,0 \\
\hline \multirow{2}{*}{ Masa muscular } & $\mathrm{Kg}$ & $28,5 \pm$ & 3,0 & $29,8 \pm 3,6$ & b $27,3 \pm$ & 3,6 & b $27,5 \pm 3,2$ & $28,2 \pm$ & 3,2 \\
\hline & $\%$ & $49,4 \pm$ & 2,6 & $46,1 \pm 4,2$ & $46,5 \pm 3$ & 3,5 & $45,7 \pm 4,1$ & $47,7 \pm$ & 3,8 \\
\hline
\end{tabular}

\section{Figura 1: Resultados según frecuencia de actividad física, distribuidos por cohorte en estudiantes} universitarios

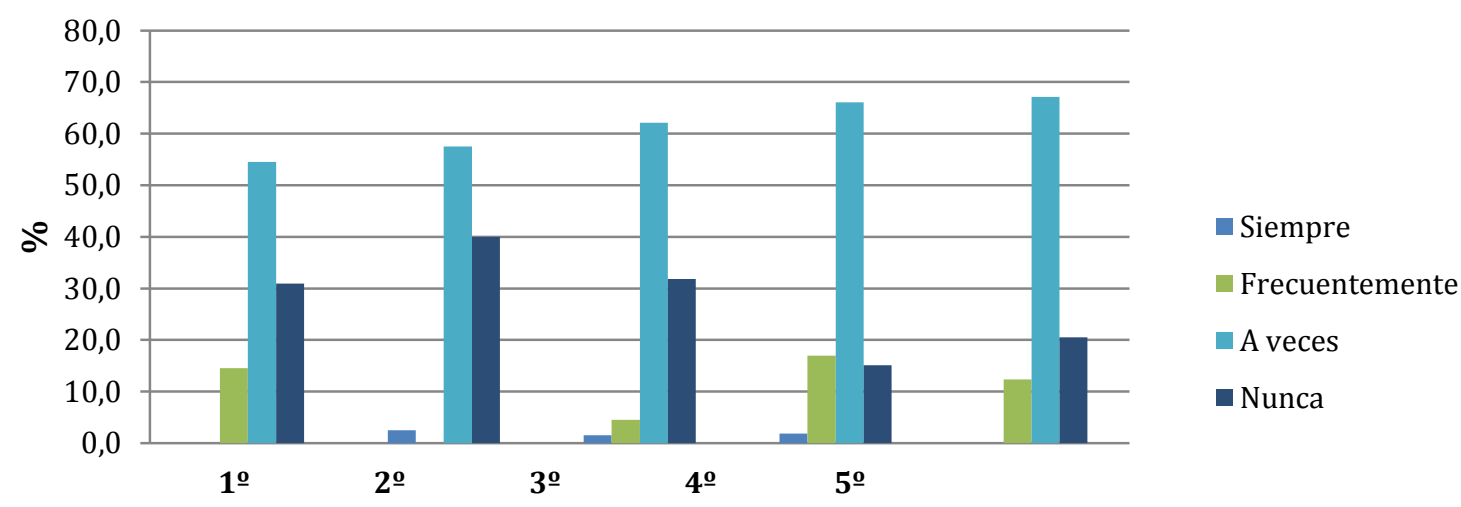


Al realizar una correlación entre los indicadores de composición corporal, el año de estudio y la actividad física para ambos sexos, se pudo determinar una baja relación inversa entre la masa grasa y actividad física $(r=-0,19)$, que indica que a mayor masa grasa, menos actividad física.
Entre la masa muscular y el año de estudio un $\mathrm{r}=$ 0,17 , que indica una ligera disminución de la masa muscular mientras más tiempo en la universidad (Tabla 3). En ambos casos el valor de "R" es más alto en los hombres.

Tabla 3: Correlación entre masa corporal, año de estudio y frecuencia de actividad física, según sexo

\begin{tabular}{lccr}
\hline Comparación de variables & \multicolumn{3}{c}{$\begin{array}{c}\text { Valor R } \\
\text { Mujeres }\end{array}$} \\
\hline IMC - Año de estudio & Total & $-0,03$ & 0,15 \\
MG - Año de estudio & 0,09 & 0,05 & 0,23 \\
MM - Año de estudio & $-0,17$ & $-0,09$ & $-0,23$ \\
IMC - Frecuencia de actividad física & 0,02 & 0,06 & $-0,17$ \\
MG - Frecuencia de actividad física & $-0,19$ & 0,02 & $-0,26$ \\
MM - Frecuencia de actividad física & 0,06 & 0,00 & 0,19 \\
\hline
\end{tabular}

MM: Masa Muscular; MG: Masa Grasa; IMC: Índice de Masa Corporal

\section{Discusión}

\section{Composición corporal}

Respecto de la composición corporal de los sujetos evaluados, no se encontró un patrón lineal claro a medida que avanzan los años, relacionado con el deterioro de la composición corporal, a excepción de una baja correlación entre masa grasa y años de estudio, y masa muscular y años de estudio en los hombres. Se aplica diferencias significativos entre $1^{\circ}$ y $2^{\circ}$ año, que se pueden explicar debido probablemente al ritmo de la vida universitaria, que implicaría cambios conductuales importantes, lo que debido a la mala organización del tiempo produciría un elevado sedentarismo que a largo plazo influiría en un cambio en la composición corporal6,12. Los resultados arrojan que al igual que en otros estudios, las mujeres presentan un mayor porcentaje de masa grasa en todos los grupos, respecto a los hombres de su misma generación. Se apreció en el grupo masculino, diferencias importantes entre los sujetos de $2^{\circ}$ año de universidad y los de 5ำ año, donde los primeros tienen una masa muscular más baja, al igual que su IMC. Espinoza et al. ${ }^{11}$, explica que puede deberse al sedentarismo que presentan los estudiantes en la etapa universitaria, tanto por la falta de espacios físicos, mala organización del tiempo o la pereza, como razón para no realizar actividad física ${ }^{7,13}$.

Un estudio prospectivo de la Universidad de Navarra, comparó el peso corporal entre estudiantes de $1^{\circ}$ y $3^{\circ}$ año, donde el $60 \%$ de los hombres aumento a diferencia de las mujeres que sólo lo hizo en un 36,8\% en el tercer año de estudio $^{14}$. Develando el impacto que tiene la vida universitaria en el peso corporal.

Otro estudio hecho en estudiantes estadounidenses que comparó a estudiantes en su primer y último años de estudios (4 años después), demostró que durante ese periodo aumentaron significativamente su peso corporal, el IMC, la masa grasa y la masa libre de la grasa ${ }^{15}$, siendo los hombres quienes presentaron un mayor aumento de estas variables.

Los hallazgos de Rangel et al., en estudiantes colombianos, señalan que el grupo etario de 21 a 25 años, presenta un sobrepeso y obesidad de $30,7 \%$ a diferencia del grupo de 18 a 20 años quienes tienen un valor de $23,8 \%{ }^{16}$. Demostrando también la influencia del tiempo universitario y edad en el aumento del peso y del IMC, que favorecen además un mayor riesgo a la salud. 
Investigadores koreanos ${ }^{17}$, han definido una relación entre el IMC y la masa muscular, no obstante la relación entre el IMC y la masa grasa puede tener mucha mayor relación en estudiantes universitarios $(r=0,81)$, considerando que el aumento de la masa grasa es debido a una ingesta nutricional inadecuada y a la falta de actividad física.

Estos cambios en la composición corporal en la vida universitaria, comienzan desde primer año. Un estudio longitudinal realizado en Bélgica, comparó los resultados de los estudiantes en la etapa escolar y luego 1,5 años después en la universidad. Los investigadores encontraron que durante este periodo aumento significativamente el peso corporal en $2 \mathrm{Kg}$ en mujeres y $4 \mathrm{Kg}$ en hombres y el IMC en ambos sexos. También disminuyó la calidad alimentaria, se duplicó el consumo de alcohol, aumentó el tiempo de estudio pero además diminuyó bruscamente el transporte activo y la participación deportiva ${ }^{18}$.

\section{Actividad Física}

Analizando las características de la Actividad física, se encontró con la reafirmación de que los hombres realizan más actividad física que las mujeres (85,3 \% y 64,9\% respectivamente), resultados similares a otros estudios en universitarios en Chile ${ }^{3,12}$.

Es importante destacar que el nivel de actividad física de los universitarios, es más alto que en la población general. En un estudio de estudiantes españoles, un 77,4\% realizaba actividad física en sus diversos niveles de intensidad ${ }^{19}$. En estudiantes de Europa se ha reportado que solo 1 de 4 estudiantes realiza actividad física que beneficie su salud ${ }^{20}$. La literatura internacional determina que la prevalencia de inactividad física varía entre $23 \%$ y $44 \%$ de acuerdo a cada país ${ }^{21}$.

El Observatorio Nacional de Salud de Italia, define que la inactividad física aumenta con la edad, siendo en las edades $18-19$ años de $27,2 \%$, en 20 24 años de $27,8 \%$ y de $25-34$ años de $34,8 \%{ }^{22}$. Pero aún, la inactividad física se asocia con el tabaquismo y una dieta no saludable ${ }^{23}$.

También se ha establecido una asociación entre la actividad física y la salud mental ${ }^{24}$, en especial en adolescentes y jóvenes adultos. Incluso se ha definido que la actividad física disminuye la depresión en un 8\% por cada hora de ejercicio adicional que se realiza por semana ${ }^{25}$. La actividad física juega un rol tan integral en la salud, que se ha descubierto que aquellos estudiantes universitarios que realizan menos de 2 horas diarias de actividad física, duplican la prevalencia de depresión y triplica los estados de ansiedad y de problemas del sueño en comparación a aquellos estudiantes que realizan más de 2 horas diarias de actividad física ${ }^{26}$.

Esta situación, sumada a la falta de programas obligatorios de actividad física, se convierte en una etapa crítica para la salud, donde se deterioran los hábitos adquiridos en la familia, facilitando aquellos poco saludables, los que a futuro, repercutirán en la salud de los estudiantes, donde el sedentarismo y la malnutrición por exceso han condicionado el incremento de las enfermedades crónicas no transmisibles ${ }^{27}$.

\section{Conclusiones}

El periodo universitario, induce a un mayor nivel de inactividad física, favoreciendo el aumento del peso corporal, IMC y masa grasa, disminuyendo la masa muscular; especialmente entre estudiantes de $1^{\underline{o}}$ y $2^{\circ}$ años de vida universitaria.

Conflicto de intereses: Ninguno declarado por los autores.

\section{Referencias}

1. Ledo-Varela MT, de Luis-Román DA, González-Sagrado M, Izaola Jauregui O, Conde-Vicente R, Aller de la Fuente R. Características nutricionales y estilo de vida en universitarios. Nutr. Hosp. 2011;26(4):814-818.

2. Olivares-C S, Lera-M L, Bustos-Z N. Etapas del cambio, beneficios y barreras en actividad física y consumo de frutas y verduras en estudiantes universitarios de Santiago de Chile. Rev. Chil. Nutr. 2008;35(1):25-35.

3. Espinoza-O L, Rodríguez-R F, Gálvez-C J, Macmillan-K N. Hábitos de alimentación y actividad física En estudiantes universitarios. Rev. Chil. Nutr. 2011;38(4) :458-65.

4. ENS. Encuesta Nacional de Salud, 2009-2010. Ministerio de Salud de Chile. 2010; Disponible en: www.redsalud.gov.cl/

5. Crovetto-M Mirta, Vio del R F. Antecedentes internacionales y nacionales de la promoción de salud 
en Chile: lecciones aprendidas y proyecciones futuras. Rev. chil. nutr. 2009; 36(1): 32-45.

6. Cossio-Bolaños MA, De Arruda M, Moyano-P A, Gañán-M E, Pino-L L, Lancho-A J. Composición corporal de jóvenes universitarios en relación a la salud. Universidad de Córdoba, España. 2011.

7. Rodríguez F, Palma X, Romo Á, Escobar D, Aragú B, Espinoza L, Gálvez J. Hábitos alimentarios, actividad física y nivel socioeconómico en estudiantes universitarios de Chile. Nutr. Hosp. 2013;28(2):447455.

8. Castillo I, Molina-García, J. Adiposidad corporal y bienestar psicológico: Efectos de la actividad física en universitarios de Valencia, España. Revista Panamericana de Salud Pública. 2009;26(4):334-340.

9. De Rose EH, Guimaraes AC. A model for optimization of somatotype in young athketes. En: Kinanthropometry II. Baltimore: Park Press; 1980.

10. Norton K, Olds T. Anthropometrica University of New South Wales Press, Sidney, Australia. 1996: 35-60.

11. Espinoza L, Rodríguez F, Gálvez J, MacMillan N. Hábitos de alimentación y actividad física en estudiantes universitarios. Rev. chil. nutr. 2011;38(4):458-465.

12. Rodríguez FJ, Espinoza LR, Gálvez J, MacMillan NG, Solis P. Estado nutricional y estilos de vida en estudiantes universitarios de la Pontificia Universidad Católica de Valparaíso. Universidad y Salud. 2013:15(2):123-35.

13. Varela M, Duarte C, Salazar I, Lema L, Tamayo J. Actividad física y sedentarismo en jóvenes universitarios de Colombia: Prácticas, motivos y recursos para realizarlas. Colomb Méd. 2011;42(3): 269-267.

14. Ruiz MNS, Ontonso IA, Armayor NC, Guillén-Grima F, de Mendoza JH, Monzo IS, Fernández BM. Modificación del peso corporal de los estudiantes universitarios en Navarra durante los tres primeros años de universidad. Nutr Hosp. 2015 31(6): 2400-2406.

15. Gropper SS, Simmons KP, Connell LJ, Ulrich PV. Changes in body weight, composition, and shape: a 4-year study of college students. Applied Physiology, Nutrition, and Metabolism. 2012;37(6):1118-1123.

16. Rangel-Caballero LG, Rojas-Sánchez LZ, GamboaDelgado EM. Sobrepeso y obesidad en estudiantes universitarios colombianos y su asociación con la actividad física. Nutr. Hosp. 2015;31(2):629-636.

17. Ko YS, You SE. Comparisons of physical fitness and body composition among Sasang types with and without body mass index as a covariate. Integrative Medicine Research. 2015;4(1):41-47.

18. Deforche B, Dyck D, Deliens T, Bourdeaudhuij I. Changes in weight, physical activity, sedentary behaviour and dietary intake during the transition to higher education: A prospective study. International Journal of Behavioral Nutrition and Physical Activity. 2015;12(1):1-10.

19. Telleria-Aramburu N, Sánchez C, Ansotegui L, Rocandio AM, Arroyo-Izaga M. Influencia de la práctica físicodeportiva sobre indicadores antropométricos y la satisfacción con el peso en hombres estudiantes universitarios: Estudio piloto. Nutr. Hosp. 2014; 31(3):1225-1231.
20. Teleman AA, Waure CD, Soffiani V, Poscia A, Pietro MLD. Physical activity and health promotion in Italian university students. Annali dell'Istituto Superiore di Sanità. 2015;51(2):106-110.

21. Haase A, Steptoe A, Sallis JF, Wardle J. Leisure-time physical activity in university students from 23 countries: associations with health beliefs, risk awareness, and national economic development. Prev Med. 2004;39(1):182-90.

22. Osservatorio Nazionale sulla Salute nelle Regioni Italiane. Rapporto Osservasalute. 2014.

23. Moreno-Gómez C, Romaguera-Bosch D, Tauler-Riera P, et al. Clustering of lifestyle factors in Spanish university students: The relationship between smoking, alcohol con- sumption, physical activity and diet quality. Public Health Nutr. 2012;15(11):2131-2139.

24. Jayakody K, Gunadasa S, Hosker C. Exercise for anxiety disorders: Systematic review. Br J Sports Med. 2014; 48(3):187-196.

25. Rothon C, Edwards P, Bhui K, Viner RM, Taylor S, Stansfeld SA, et al. Physical activity and depressive symptoms in adolescents: a prospective study. BMC Med. 2010;8(1):1.

26. Wu X, Tao S, Zhang Y, Zhang S, Tao F. Low physical activity and high screen time can increase the risks of mental health problems and poor sleep quality among Chinese college students. PLoS ONE. 2015;10(3): e0119607.

27. Durán S, Castillo M, Vio del R F. Diferencias en la calidad de vida de estudiantes universitarios de diferente año de ingreso del campus antumapu. Rev. chil. nutr. 2009;3(3):200-209. 